\title{
Retinal arterial macroaneurysms: risk factors and natural history
}

\author{
Robert W Panton, Morton F Goldberg, Marilyn D Farber
}

\begin{abstract}
A case control study was conducted to identify the systemic and ocular risk factors for retinal arterial macroaneurysms. Forty-three patients with 52 photographically confirmed macroaneurysms were located. Forty-three agematched, race-matched concurrent control patients were also identified. The patients with macroaneurysms had decreased visual acuity $(p<0.0001)$ and a higher prevalence of hypertension $(p=0.037)$, female sex $(p=0.099)$, and retinal vein occlusions $(p=0.055)$ than controls. In patients with both a macroaneurysm and venous occlusion there was a 12.0 times higher prevalence of macroaneurysms in the area of retina drained by the occluded vein $(p<0.05)$. Common findings associated with macroaneurysms included retinal haemorrhage ( $81 \%$ of patients), retinal exudate $(70 \%)$, vitreous haemorrhage (30\%), macular involvement $(30 \%)$, and distal arteriolar narrowing (26\%). Arteriolar occlusion occurred spontaneously $(8 \%)$ or after laser photocoagulation (16\%).
\end{abstract}

Retinal arterial macroaneurysms are acquired dilatations of the retinal arterioles, usually occurring in the first three orders of the arteriolar tree.' Previous work has demonstrated a strong association between the development of macroaneurysms and several systemic factors. Most patients are over 60 years of age, ${ }^{12}$ and $60-80 \%$ are women. ${ }^{2-4}$ The large majority of patients have hypertension $^{3-5}$ and generalised atherosclerotic disease. ${ }^{36}$ Other reported associations include hyperlipidaemia, ${ }^{46}$ polycythaemia,${ }^{2}$ and cerebrovascular disease. ${ }^{467}$

No ocular risk factor for arterial macroaneurysms has been definitively confirmed. However, macroaneurysms have been reported in association with several diseases of the retinal vasculature, including retinal telangiectasia, ${ }^{8}$ arterial emboli, ${ }^{47}$ and retinal vein occlusion. ${ }^{2469}$

Although many patients with macroaneurysms have been reported and several risk factors suggested, there has been no controlled study of risk factors. We therefore conducted a case control study to elucidate the ocular and systemic risk factors for the development of arterial macroaneurysms. We also investigated the natural history of macroaneurysms.

\section{Methods and materials}

We reviewed the computerised diagnostic records from 1971 to 1988 of the Ophthalmic Photography Department of the University of Illinois at Chicago. A total of 43 patients with 52 arterial macroaneurysms were identified. The diagnosis was confirmed in every case with fundus photography demonstrating round, saccular, or fusiform dilatation of the retinal arterioles to greater than $100 \mu \mathrm{m}$. Intravenous fluorescein angiography was performed on $98 \%$ $(42 / 43)$ of the patients.

Control patients were obtained for the study by review of random groups of 50 outpatient ophthalmic medical records to identify 43 control patients who were matched to the cases for the following variables: race, age within five years, and date of examination within three years. After control patients had been identified, the ipsilateral eye of cases and controls were matched.

A standardised history and physical examination form was used for new clinic patients, and data from cases and controls were obtained primarily from those forms. In patients with large vitreous haemorrhages, refraction and funduscopy were not performed at the initial examination but at subsequent examinations. Any case with missing data was included overall, but the case and corresponding control were excluded from analysis of the specific variable.

Hypertension was identified by prior diagnosis, by the use of systemic hypotensive medications, or by a blood pressure reading of over $140 / 90 \mathrm{mmHg}$ at the time of examination. ${ }^{111}$ Glaucoma was defined by history, by the use of ocular hypotensive drugs, or by the presence of raised intraocular pressure with characteristic optic nerve and visual field changes. ${ }^{10}$ Other diseases, such as heart disease and diabetes, were identified by prior diagnosis or use of appropriate medications.

Statistical analysis was performed with the computerised statistical program True Epistat. Significance was tested with the $\chi^{2}$ or Student's $t$ test where appropriate. Two-tailed analyses were performed for all parameters except vision. All visual acuity data were converted to LogMAR scores $^{12}$ before a one-tailed Student's $t$ test was performed. As a measure of the relative risk of getting a macroaneurysm with a potential risk factor present, odds ratios with Cornfield's $95 \%$ confidence limits were calculated.

\section{Results}

All cases were matched with controls for age, race, the eye (Table 1). The mean age was $69 \cdot 7$ years (range, 31-92 years) in the cases and 69.3 years in the controls. The racial distribution was the same in the cases and controls: 28 blacks, nine whites, and six Hispanics. The left eye was more commonly affected (63\%), but the difference was not statistically significant $(p>0.05)$.

A comparison of cases and controls by ocular and medical history is shown in Table 2 . Hyper- 
Table 1 Matched demographic variables in 43 case control pairs

\begin{tabular}{lcc}
\hline Matched variable & Cases & Controls \\
\hline Age (mean years) & $69 \cdot 7$ & $69 \cdot 3$ \\
Race: black & 28 & 28 \\
white & 9 & 9 \\
Hispanic & 6 & 6 \\
Eye: right & 16 & 16 \\
$\quad$ left & 27 & 27 \\
\hline
\end{tabular}

tension was present in 34 of 43 cases but in only 24 of 43 controls $(p=0.037)$. The odds ratio $(O R)$ for hypertension was 2.99 , with $95 \%$ confidence limits of $1 \cdot 1$ and $8 \cdot 7$. Women were more prevalent in the cases than controls $(O R=2.47)$ but with only marginal significance $(p=0.099)$. Heart disease was also more common in the cases $(\mathrm{OR}=2 \cdot 01)$, but the difference was not statistically significant $(p=0 \cdot 175)$. No association was found with the history of strabismus, glaucoma, arthritis, kidney disease, diabetic retinopathy, or diabetes. Analysis by stepwise logistic regression did not significantly affect the results.

The ipsilateral eye of cases and controls was matched for analysis of the ocular examination variables in Table 3 . The mean best corrected visual acuity was $20 / 160$ in the affected eye of the cases and 20/50 in the ipsilateral eye of the controls $(p<0.0001)$. There were no significant differences between cases and controls for horizontal cup-to-disc ratio, intraocular pressure, or spherical equivalent.

Cases and controls had similar prevalences of strabismus, lens opacities, drusen, diabetic retinopathy, and corneal disease (Table 4). Retinal vein occlusion occurred in five of the cases but in none of the controls $(p=0.055)$.

Table 2 Ocular and medical history of case control pairs

\begin{tabular}{|c|c|c|c|c|c|}
\hline Historical variable & Cases & Controls & p Value & $\begin{array}{l}\text { Odds } \\
\text { ratio }\end{array}$ & $\begin{array}{l}95 \% \text { confidence } \\
\text { interval }\end{array}$ \\
\hline Hypertension & $34 / 43$ & $24 / 43$ & 0.037 & 2.99 & $(1 \cdot 1-8 \cdot 7)$ \\
\hline Female sex & $34 / 43$ & $26 / 43$ & 0.099 & $2 \cdot 47$ & $(0 \cdot 86-7 \cdot 2)$ \\
\hline Heart disease & $20 / 41$ & $13 / 41$ & $0 \cdot 175$ & $2 \cdot 01$ & $(0 \cdot 76-5 \cdot 4)$ \\
\hline Strabismus & $1 / 39$ & $5 / 39$ & $0 \cdot 200$ & $0 \cdot 18$ & $(0 \cdot 02-1 \cdot 7)$ \\
\hline Glaucoma & $2 / 40$ & $5 / 40$ & 0.432 & 0.37 & $(0.07-2 \cdot 4)$ \\
\hline Arthritis & $20 / 38$ & $21 / 38$ & 1.000 & 0.90 & $(0 \cdot 33-2 \cdot 4)$ \\
\hline Kidney disease & $2 / 38$ & $1 / 38$ & 1.000 & 2.06 & $(0 \cdot 18-23)$ \\
\hline Diabetic retinopathy & $4 / 43$ & $3 / 43$ & 1.000 & $1 \cdot 37$ & $(0 \cdot 24-8 \cdot 4)$ \\
\hline Diabetes & $7 / 41$ & $7 / 41$ & 1.000 & 1.00 & $\mathrm{NC}^{\star}$ \\
\hline
\end{tabular}

${ }^{\star} \mathrm{NC}=$ not calculable.

Table 3 Mean values of ocular examination in case control pairs

\begin{tabular}{llccc}
\hline Examination variable & Number of pairs & Cases & Controls & p Value \\
\hline Visual acuity & 43 & $20 / 160$ & $20 / 50$ & $<0.0001$ \\
Horizontal cup-to-disc ratio & 39 & 0.38 & 0.34 & $0 \cdot 19$ \\
Intraocular pressure (mmHg) $_{\text {Spherical equivalent }}^{\star}$ & 43 & 16 & 17 & 0.28 \\
& 30 & +0.86 & +0.51 & 0.31 \\
\hline
\end{tabular}

^Eleven aphakic and pseudophakic individuals were excluded from calculations of spherical equivalents.

Table 4 Prevalence of associated ocular disorders identified by examination of case control pairs

\begin{tabular}{lrllll}
\hline Historical variable & Cases & Controls & $p$ Value & $\begin{array}{l}\text { Odds } \\
\text { ratio }\end{array}$ & $\begin{array}{l}\text { 95\% confidence } \\
\text { interval }\end{array}$ \\
\hline Retinal vein occlusion & $5 / 43$ & $0 / 43$ & 0.055 & $\mathrm{NC}$ & $\mathrm{NC}$ \\
Strabismus & $3 / 43$ & $5 / 43$ & $0 \cdot 356$ & $0 \cdot 57$ & $(0 \cdot 10-3 \cdot 0)$ \\
Lens opacity & $11 / 43$ & $7 / 43$ & $0 \cdot 427$ & $1 \cdot 77$ & $(0 \cdot 55-5 \cdot 8)$ \\
Drusen & $6 / 43$ & $4 / 43$ & $0 \cdot 738$ & $1 \cdot 58$ & $(0 \cdot 36-7 \cdot 4)$ \\
Diabetic retinopathy & $5 / 43$ & $4 / 43$ & 1.000 & $1 \cdot 28$ & $(0 \cdot 27-6 \cdot 3)$ \\
Corneal disease & $2 / 43$ & $1 / 43$ & $1 \cdot 000$ & $2 \cdot 05$ & $(0 \cdot 19-23)$ \\
\hline
\end{tabular}

$\star \mathrm{NC}=$ not calculable
Table 5 Clinical characteristics of 43 arterial macroaneurysms

\begin{tabular}{lrr}
\hline Characteristic & Number of patients & $\%$ \\
\hline Number of macroaneurysms: & 37 & \\
One & 5 & 86 \\
Two & 1 & 12 \\
Five & 22 & 2 \\
Location of macroaneurysms: & 12 & 51 \\
Superotemporal & 3 & 28 \\
Inferotemporal & 0 & 7 \\
Superonasal & 6 & 0 \\
Inferonasal & 12 & 14 \\
Off optic nerve & 22 & 28 \\
Order of retinal artery: & 5 & 51 \\
First & 3 & 12 \\
Second & 1 & 7 \\
Third & Fourth & 2 \\
Fifth & & \\
\hline
\end{tabular}

Detailed ophthalmoscopic examination was made of the five patients with both a macroaneurysm and venous occlusion. For example, in case 1 and in a non-detailed case the macroaneurysm followed the branch retinal vein occlusion in the same retinal quandrant three years and fourteen years later, respectively. In two other cases the macroaneurysm and retinal vein occlusion occurred in the same retinal quadrant, but the temporal relationship is unknown. The fifth patient had a hemiretinal vein occlusion and a macroaneurysm in different retinal areas. Overall the five patients with both a macroaneurysm and venous occlusion had a $12 \cdot 0$ times higher prevalence of macroaneurysms in the retinal area supplied by the occluded vein than in the remaining retinal area $(p<0.05)$.

The characteristics of the macroaneurysms are shown in Table 5 . A total of $86 \%$ of the patients presented with one macroaneurysm, $12 \%$ had two, and $2 \%$ had five. All 43 cases presented with unilateral macroaneurysms, but two patients had subsequent involvement of the other eye. Macroaneurysms usually presented superotemporally $(51 \%)$ or inferotemporally $(28 \%)$ and often occurred on the first three orders of the arteriolar tree. Most macroaneurysms were surrounded by retinal haemorrhage (81\%) and exudate $(70 \%)$. Macular exudate or haemorrhage $(30 \%)$, vitreous haemorrhage $(30 \%)$, distal arteriolar narrowing (26\%), and arterial emboli (9\%) were also observed.

Branch artery occlusion occurred spontaneously in two out of 24 individuals (8\%) and after laser therapy in three out of 19 individuals $(16 \%)$. Treatment of nine patients with argon laser photocoagulation directly to the macroaneurysm and surrounding retina resulted in two arteriolar occlusions. Direct aneurysmal treatment with dye yellow laser photocoagulation in five patients resulted in one arteriolar occlusion (case 1). No occlusions were seen in three patients treated with only perianeurysmal argon laser photocoagulation. None of the five branch artery occlusions had a major effect on visual acuity.

\section{Case histories}

CASE 1

A 63-year-old black female with a 15-year history of adult onset diabetes and systemic hyperten- 


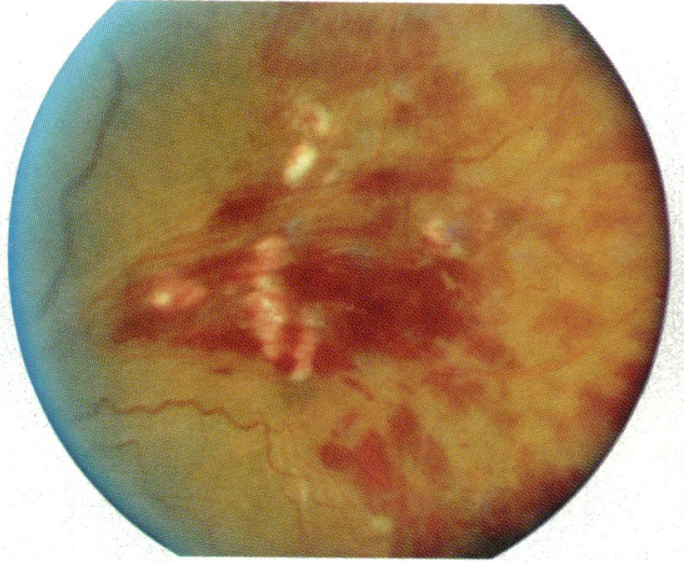

Figure 1A

sion complained of blurred vision in her left eye. Ocular examination demonstrated a best corrected visual acuity of $20 / 40$ and a left superotemporal branch retinal vein occlusion (Fig 1A). Fluorescein angiography showed dilated retinal veins and large areas of hypofluorescence (Fig 1B). Two years later the haemorrhage cleared (Fig 1C), and the visual acuity returned to 20/20. The angiogram demonstrated capillary nonperfusion and remodelling (Fig 1D). Arteriolar irregularity was seen at the origin of dilated collateral vessels (arrow).

At the same location one year later there was a large arterial macroaneurysm with surrounding haemorrhage and exudate (Figs 1E, 1F). Because the exudate had encroached on the fovea and the visual acuity had dropped to $20 / 40$, the patient received dye yellow laser photocoagulation (three applications, $577 \mathrm{~nm}$ wavelength, $600 \mu \mathrm{m}$ spot size, $0.5 \mathrm{~s}$ duration, $170 \mathrm{~mW}$ intensity) directly to the macroaneurysm, sparing the feeding and draining segments of the arteriole. ${ }^{13-15}$

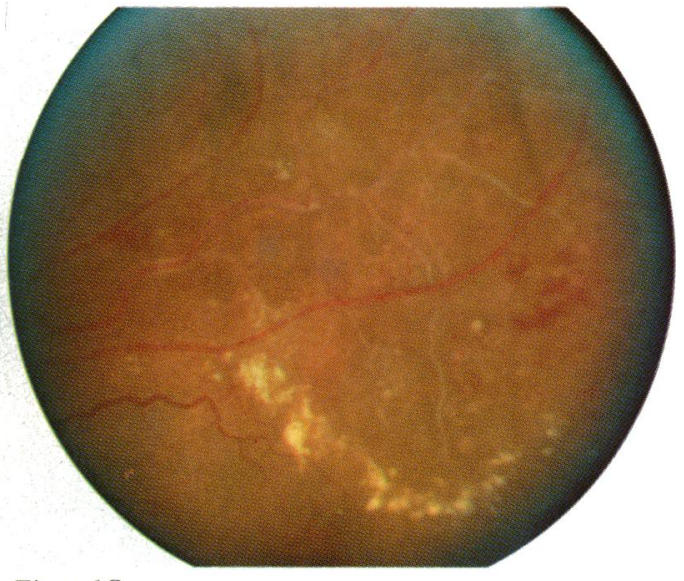

Figure 10

Two months after photocoagulation the foveal exudate increased, and the visual acuity dropped to $20 / 200$. But six months later the haemorrhage and exudate resorbed, leaving a visual acuity of 20/25 (Fig 1G). The angiogram showed occlusion of the arteriole just proximal to the site of photocoagulation and retrograde filling of the distal arteriole through collateral vessels (Fig $1 \mathrm{H})$.

\section{CASE 2}

A healthy 66-year-old white male presented with a best corrected visual acuity of 20/40 in the left eye, with two macroaneurysms of the superotemporal artery (Fig 2A). The second macroaneurysm was fibrotic and surrounded with haemorrhage and exudate. No laser photocoagulation was recommended. Two months later the proximal macroaneurysm obstructed, resulting in ischaemic swelling of the retina (Fig 2B) and a visual acuity of 20/60. Non-perfusion of the distal arteriolar segment was demonstrated with

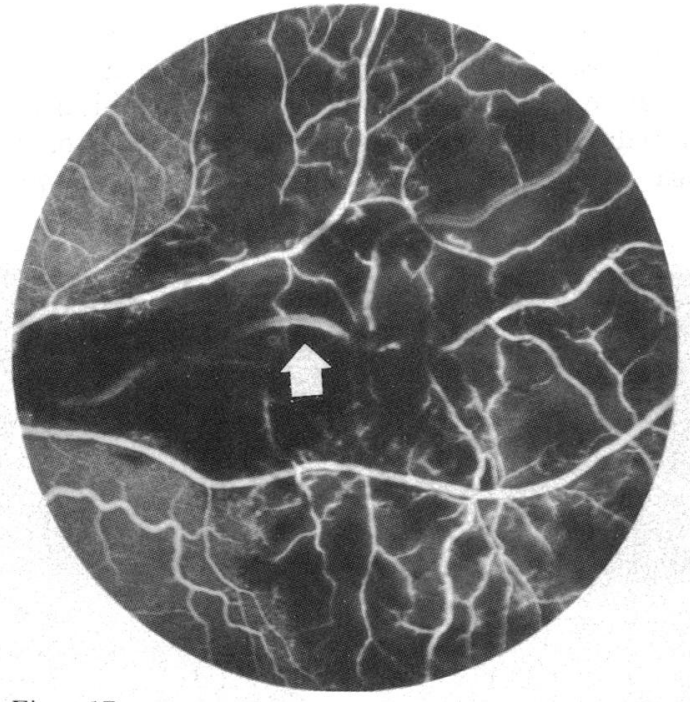

Figure $1 B$

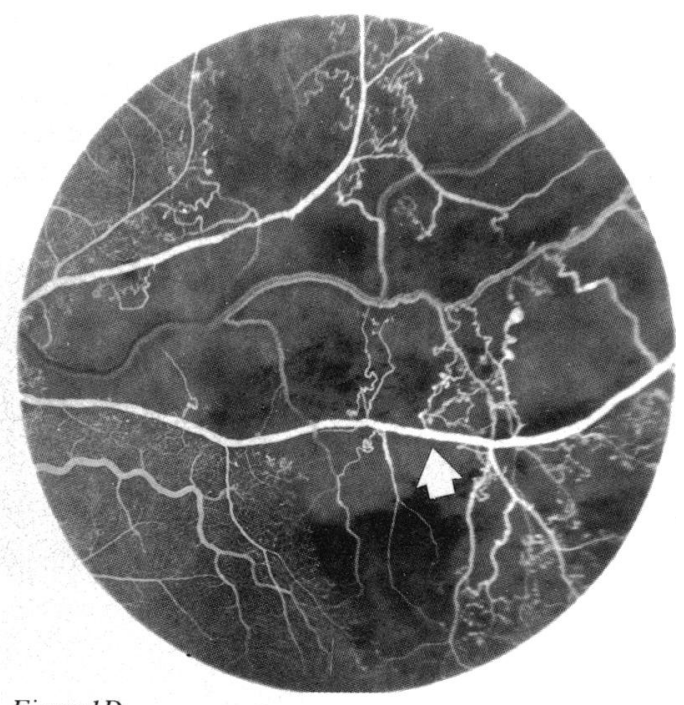

Figure 1D

Figure 1 A: Left superotemporal branch retinal vein occlusion. B: Corresponding fluorescein angiogram demonstrates a dilated retinal vein (arrow) and large areas of hypofluorescence. $C:$ Two years later the haemorrhage has cleared, revealing lipid exudate, dilated capillaries, and sclerotic veins. D: Fluorescein angiography shows capillary non-perfusion and remodelling. Arteriolar irregularity is seen at the origin of dilated collateral vessels (arrow). E: One year later the same site has a large arterial macroaneurysm with surrounding haemorrhage and exudate. F: Angiography shows discrete filling of the macroaneurvsm. $G:$ Eight months after photocoagulation the haemorrhage resorbed, leaving foveal exudate. H: Fluorescein angiography demonstrates occlusion of the arteriole (arrow) just proximal to the site of photocoagulation. There is retrograde filling of the distal
arteriole. 


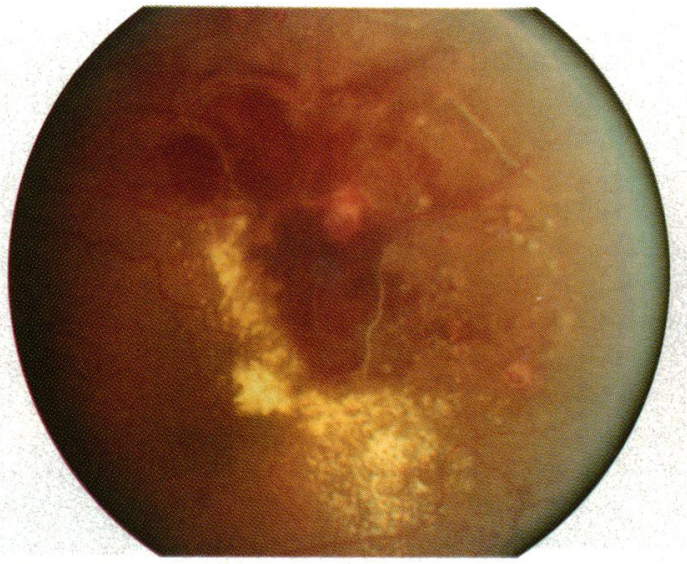

Figure $1 E$

fluorescein angiography (Fig 2C). Within six months both macroaneurysms had involuted, and the visual acuity returned to $20 / 40$.

\section{Discussion}

Several studies have described the clinical characteristics of patients with macroaneurysms, but the patients were not compared with a control population..$^{3-815-18}$ We therefore undertook a case control study to identify the systemic and ocular risk factors for the development of macroaneurysms.

Arterial macroaneurysms are more common in elderly patients. The mean age of the cases in our study was 69.7 years. Histopathologically, aging of arterioles is characterised by an increase in intimal collagen and a replacement of medial muscle fibres with collagen. ${ }^{219}$ The arterial wall becomes less elastic and more susceptible to dilatation from elevated hydrostatic pressure. ${ }^{3}$

Systemic hypertension and haemodynamic stress have been implicated in the pathogenesis of intracranial aneurysms. ${ }^{20}$ Hypertension is also a significant risk factor for retinal arterial macroaneurysms, and $79 \%$ of our cases were hypertensive. Hypertension can lead to hyaline degeneration of vessel walls, loss of autoregulatory tone, and arterial dilatation. ${ }^{21}$ Hypertensive patients also have raised hydrostatic pressures. According to the Laplace equation, a raised

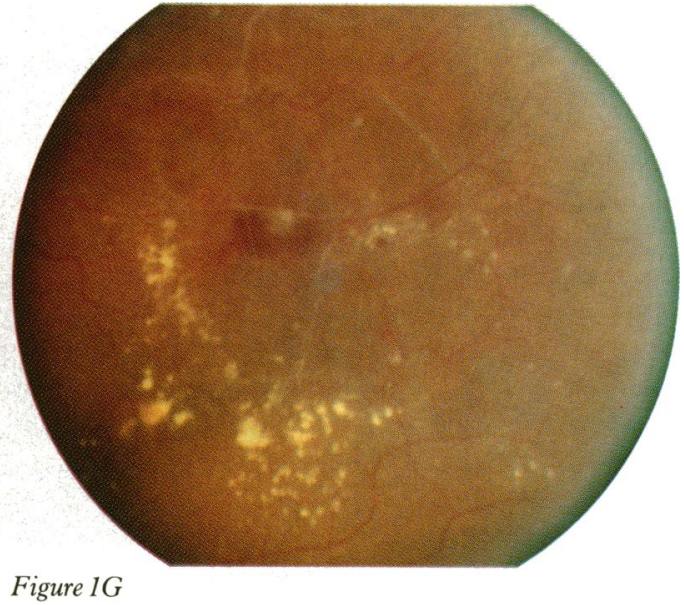

transmural pressure is directly proportional to a raised wall tension, which might predispose to macroaneurysm formation..$^{20}$

Venous occlusion was more common in the patients with macroaneurysms. A statistical association between the two diseases may be explained by several possibilities: ( $a$ ) Both diseases may have similar risk factors, such as age and hypertension. ${ }^{11}$ (b) Venous occlusion may be caused by an adjacent macroaneurysm. ${ }^{2}$ $\operatorname{Or}(c)$ a macroaneurysm may result from venous occlusion. The last possibility could occur when chronic venous stasis leads to local arterial thrombosis, endothelial damage, and aneurysm formation. ${ }^{7}$ Alternatively, a macroaneurysm following venous occlusion could form at the origin of a dilated collateral vessel, as occurred in case 1. A macroaneurysm might arise at such a location because of arterial wall weakness or local turbulent flow. ${ }^{2718}$ Although the present study does not distinguish among these possibilities, the apparently common occurrence of macroaneurysms and venous occlusions in the same retinal area suggests the influence of local factors.

Females were more prevalent among cases $(79 \%)$ than controls $(60 \%)$, but the relatively small sample size was insufficient to document statistical significance. Several series have previously reported that women represent the large majority of patients with macroaneurysms;

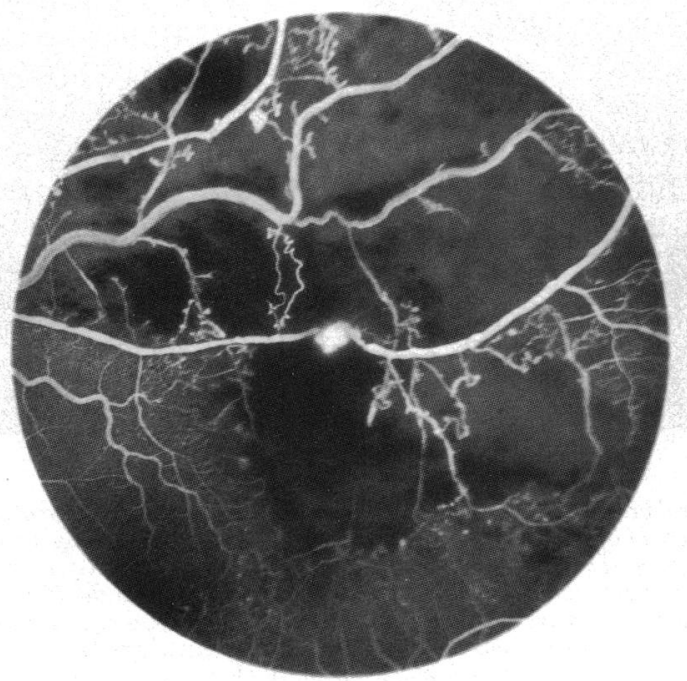

Figure $1 F$

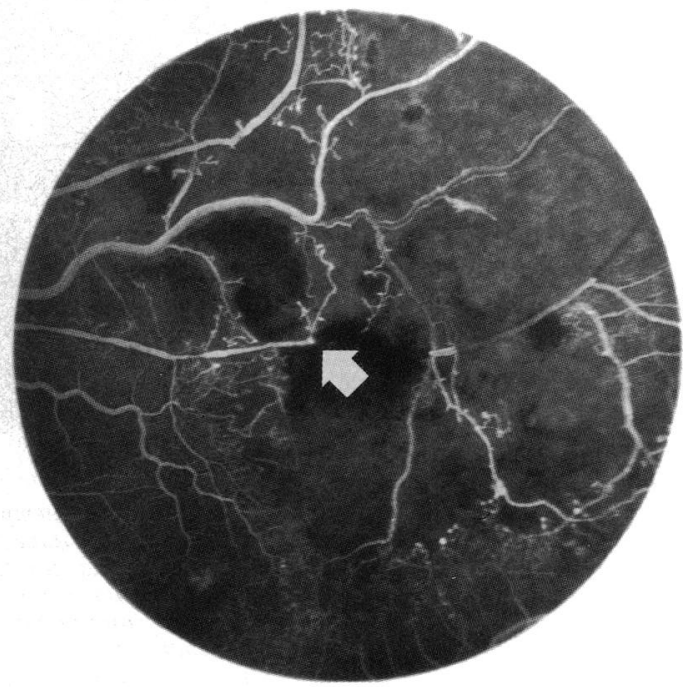

Figure $1 \mathrm{H}$ 


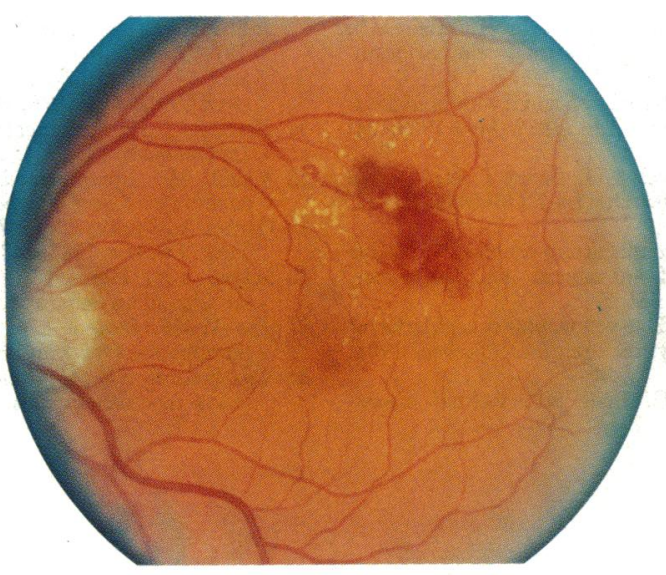

Figure 2A



Figure $2 B$

Figure 2 A: Left eye, two superotemporal arterial macroaneurysms. The distal macroaneurysm is fibrotic and surrounded with haemorrhage and exudate. B: Two months later obstruction of the proximal macroaneurysm leads to ischaemic swelling of the retina. C: Angiography demonstrates distal arteriolar non-perfusion.

however, the significance of the observation is uncertain because no control groups were provided. ${ }^{2-8} 151619$ Intracranial saccular aneurysms, particularly those on the internal carotid artery, are also more common among women. ${ }^{20}$ This female preponderance has been attributed to both heritable and hormonal causes. ${ }^{20}$

Our retrospective analysis was limited to a standardised history and physical examination form, and we were unable to analyse potential risk factors such as cerebrovascular disease, atherosclerosis, polycythaemia, and hyperlipidaemia. Our study was also unable to evaluate microvascular risk factors such as arterial emboli and plaques in a controlled fashion, because only the cases underwent fluorescein angiography. Despite these inherent limitations, our study showed that patients with arterial macroaneurysms also have an increased prevalence of other vascular changes, both systemically (for example, hypertension and aging) and locally (for example, retinal vein occlusions).

In addition to examining risk factors we began a preliminary study of the natural history and therapy of macroaneurysms. Arteriolar occlusion was seen spontaneously in $8 \%$ of patients and after laser photocoagulation in $16 \%$

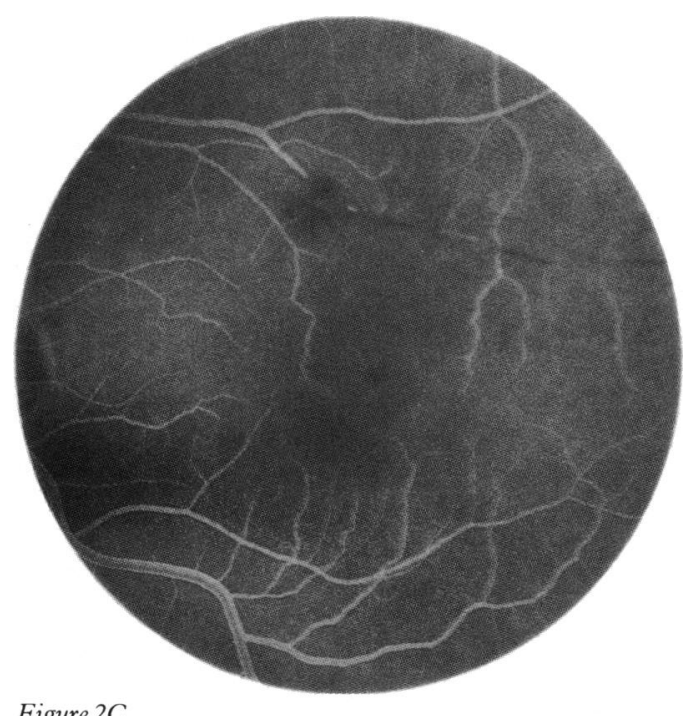

of patients. No occlusions were seen after exclusively perianeurysmal laser treatment. Several authors have suggested that arteriolar obstruction may be more common after direct aneurysmal rather than perianeurysmal photocoagulation. ${ }^{16} 1722$

In the largest series of patients to receive dye yellow laser photocoagulation for macroaneurysms Joondeph and colleagues reported that no arterial occlusions occurred after treatment of 12 patients. ${ }^{15}$ However, our case 1 had an arterial occlusion after dye yellow photocoagulation with an identical treatment protocol. Arterial occlusions may not be readily apparent by ophthalmoscopy, possibly because of distal collateral flow. Fluorescein angiography after laser treatment of macroaneurysms might reveal a higher incidence of branch artery occlusions than previously believed.

To follow up our retrospective analysis of macroaneurysm risk factors we are now prospectively observing our patients with photography and angiography to determine the rates of involution of macroaneurysms, resorption of haemorrhage and exudate, and occlusion of arterioles.

The authors thank Mr Norbert Jednock and the Ophthalmic Photography Department for their assistance. This work was supported in part by training grant 7038 and core grant 1792 from the National Eye Institute, Bethesda, Maryland, and by unrestricted grants from the Alcon Research Institute, Fort Worth, Texas, and Research to Prevent Blindness, Inc, New York, New York.

1 Robertson DM. Macroaneurysm of the retinal arteries. Ophthalmology 1973; 77: 55-67.

2 Lavin MJ, Marsh RJ, Peart S, Rehman A. Retinal arterial macroaneurysms: a retrospective study of 40 patients. $\mathrm{Br} f$ Ophthalmol 1987; 71: 817-25.

3 Rabb MF, Gagliano DA, Teske MP. Retinal arterial macro aneurysms. Surv Ophthalmol 1988; 33: 73-96.

4 Palestine AG, Robertson DM, Goldstein BG. Macroaneurysms of the retinal arteries. Am $\mathcal{F}$ Ophthalmol 1982; 93: 164-71.

5 Abdel-Khalek MN, Richardson J. Retinal macroaneurysm natural history and guidelines for treatment. $B r$, Ophthalmol 1986; 70: 2-11.

6 Cleary PE, Kohner EM, Hamilton AM, Bird AC. Retina macroaneurysms. Brf Ophthalmol 1975; 59: 355-61.

7 Lewis RA, Norton EWD, Gass JDM. Acquired arteria macroaneurysms of the retina. Br $\mathcal{F}$ Ophthalmol 1976; 60 : 21-30.

8 Asdourian GK, Goldberg MF, Jampol L, Rabb MF. Retina macroaneurysms of the retina. Br 7 Ophthalmol 1976; 60 ; 21-30.

9 Panton RW, Goldberg MF. Risk factors of retinal arterial macroaneurysm. Invest Ophthalmol Vis Sci (ARVO abstracts) 1989; 30 (suppl): 476 
10 Johnston RL, Brucker AJ, Steinmann W, Hoffman ME, Holmes JH. Risk factors of branch retinal vein occlusion. Arch Ophthalmol 1985; 103: 1831-2

11 Apiah AP, Trempe CL. Risk factors associated with branch vs. central retinal vein occlusion. Ann Ophthalmol 1989; 21 : 153-7.

12 Ferris FL, Kassoff A, Bresnick GH, Bailey I. New visual acuity charts for clinical research. Am $\mathcal{F}$ Ophthalmol 1982, 94: $91-6$.

13 Russell SR, Folk JC. Branch retinal artery occlusion after dye yellow photocoagulation of an arterial macroaneurysm. Am F Ophthalmol 1987; 104: 186-7.

14 Mainster MA, Whitacre MM. Dye yellow photocoagulation of retinal arterial macroaneurysms. Am f Ophthalmol 1988 105: $97-8$.

15 Joondeph BC, Joondeph HC, Blair NP. Retinal macro- aneurysms treated with the yellow dye laser. Retina $1989 ; 9$.

16 Nadel AJ, Gupta KK. Macroaneurysm of the retinal arteries. Arch Ophthalmol 1976; 94: 1092-6.

17 Francois J. Acquired macroaneurysm of the retinal arteries. Int Ophthalmol 1979; 1: 153-61.

18 Khalil M, Lorenzetti DWC. Acquired retinal macroaneurysms. Can $\mathcal{F}$ Ophthalmol 1979; 14: 163-9.

19 Garner A, Klintworth G. Pathobiology of ocular disease. New York: Dekker, 1982: 1480-525.

20 Sekhar LN, Heros RC. Origin, growth, and rupture of saccular aneurysms: a review. Neurosurgery 1981; 8: 248-60. 21 Tso MOM, Jampol LM. Pathophysiology of hypertensive retinopathy. Ophthalmology 1982; 89: 1132-45.

22 Van Nouhuys E, Deutman AF. Argon laser treatment of retinal macroaneurysms. Int Ophthalmol 1980; 2: 45-53. 